\title{
Evaluation of MAV/UAV Collaborative Combat Capability Based on Network Structure
}

\author{
Jieru Fan, ${ }^{1,2}$ Dongguang $\mathrm{Li}\left(\mathbb{D},{ }^{1,2}\right.$ and Rupeng $\mathrm{Li}^{1,2}$ \\ ${ }^{1}$ Science and Technology on Electromechanical Dynamic Control Laboratory, China \\ ${ }^{2}$ Department of Mechatronical Engineering, Beijing Institute of Technology, Beijing 100081, China \\ Correspondence should be addressed to Dongguang Li; lidongguang@bit.edu.cn
}

Received 17 May 2018; Accepted 21 October 2018; Published 5 December 2018

Academic Editor: Giovanni Palmerini

Copyright ( 2018 Jieru Fan et al. This is an open access article distributed under the Creative Commons Attribution License, which permits unrestricted use, distribution, and reproduction in any medium, provided the original work is properly cited.

\begin{abstract}
The collaborative combat of manned/unmanned aerial vehicles (MAVs/UAVs) is a popular topic in combat application research. It maximizes the autonomous combat capability of UAVs and the control capability of MAVs. Furthermore, it improves the comprehensive combat effectiveness. The quantitative description of intercommunication in different aircrafts along with the evaluation of the collaborative combat capability is an emphasis in military research. This paper analyzes the collaborative combat process. Node and edge models are established in the MAV/UAV collaborative network. The intercommunication and combat behaviors among combat entities are analyzed. Based on the information entropy, the effect of capability uncertainties on the collaborative combat is described quantitatively. An evaluation method of the MAV/UAV collaborative combat capability is proposed. Finally, an example is given to demonstrate the proposed model and evaluation method that prove its feasibility and effectiveness.
\end{abstract}

\section{Introduction}

With the development of aviation technology and the increasing military demands, the research and development (R\&D) of unmanned aerial vehicles (UAVs) has gradually developed trends of generalization and serialization [1]. The role of UAVs on the battlefield has gradually transitioned from reconnaissance to aerial battle [2]. However, because the intelligent systems of UAVs cannot replace human thinking and judgment, UAVs cannot easily perform complex combat missions independently [3]. Therefore, a mode of collaborative combat of manned/unmanned aerial vehicle (MAV/UAV) has emerged.

The collaborative combat systems of MAV/UAV make full use of the advantages of UAVs, such as their low cost, zero life risk, strong maneuverability, and powerful survival ability [4], which compensate for their disadvantages, such as their low intelligence and weak ability in handing emergencies. Collaborative combat systems reduce the cost of using manned aircrafts to execute dangerous tasks and changes in air combat patterns. The collaborative combat capability of MAV/UAV in systemic confrontation has been significantly improved in the information age.

MAV/UAV collaborative systems are becoming a new area of research in the field of aviation, but experts generally concentrate on the equipment itself. For future air combat, relevant research results about how to determine index systems of MAV/UAV collaborative combat and evaluate their combat capability have not been found.

Developing a reasonable model and description of the collaborative combat architecture is the primary task for collaborative combat systems. With the deepening knowledge of equipment systems, the Department of Defense Architecture Framework (Do DAF) $[5,6]$, proposed by the US Army, has been unable to reflect the complexity, connectivity, and networking features of the combat process. To remedy this limitation, experts have explored new methods to make the model more reasonable. Boyd proposed an OODA model 
[7] that regards the combat process as a dynamic cycle consisting of the following four links: observe, orient, decide, and act. Based on this model, Cares established a static model $[8,9]$. Military equipment were abstracted into four types of network nodes, and the transmissions of information, material, and energy in the battle process were expressed by the edges. By combining the complex network with the agent method, Dekker extended the traditional network method and proposed a C4ISR model [10], showing that the use of complex networks to study combat processes could improve decision-making. The model not only determined the key elements in the combat cycle but also improved the communication and workflow of the combat systems. Tolk et al. [11] improved the information age model established by Cares but did not consider the impact of node performance on combat effectiveness $[12,13]$. Most current research on capability assessments is based on the model of a traditional index system $[14,15]$, which is decomposed by a hierarchy analysis process. Such research ignores the influence of interactions among entities on the capability of the overall collaborative combat systems.

The advancement of information technology has improved the strength of the interactive relationship between military equipment, which has a significant impact on modern warfare. This improvement was motivated by the consideration that the simple superposition of the equipment performances alone cannot adequately evaluate the ability of a whole system. Therefore, when representing the combat capability of the systems, the coordination relationship among the military equipment must be considered. In particular, during the MAV/UAV collaborative combat process, different types of aircraft have great discrepancies in flight speed, information transmission, and applicable combat tasks, which form a heterogeneous feature. The heterogeneous node integration and collaborative combat capability evaluation are not discussed in the literatures.

When studying collaborative combat systems, the primary task is to reasonably model and describe the collaborative combat architecture. The systems are used to evaluate the MAV/UAV collaborative combat capability via the construction of reasonable indexes or the adoption of effective methods and to locate the flaws and deficiencies of the combat capability. The results can be used as a theoretical basis to optimize the architecture to effectively improve the combat capability of the entire system.

In summary, for a deeper understanding of the MAV/ UAV collaborative combat systems, this paper describes the information transmission relationship among military equipment. Based on the operation loop, network node and edge models of the MAV/UAV collaborative combat systems are established and analyzed. The uncertainty influence on the capability of the collaborative combat is measured according to information entropy $[16,17]$. A new method for evaluating the MAV/UAV collaborative combat capability is proposed. Research on the modeling and capability evaluation methods of MAV/UAV collaborative combat systems is the cornerstone of research on the complete collaborative combat systems. The research provides a quantitative decision basis for military R\&D and the procurement of

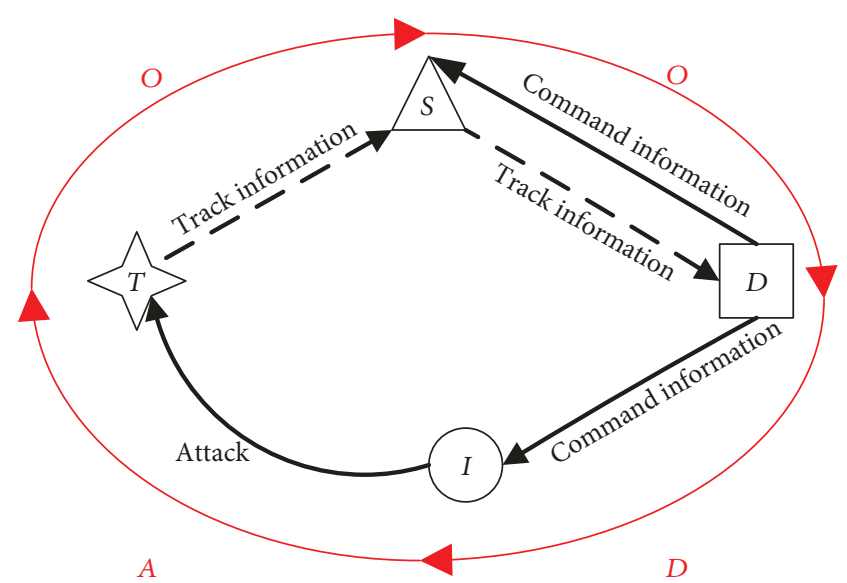

$\begin{array}{ll}\text { St Target node } & D \text { Decision maker node } \\ \text { S Sensor node } & \text { I Influencer node }\end{array}$

Figure 1: Network nodes.

new aircraft types. Moreover, it delivers a reliable basis for the $R \& D$ of the combat guiding ideology and tactics.

\section{Network Model of MAV/UAV Collaborative Combat}

Establishing a network model is a precondition for studying MAV/UAV collaborative combat systems. According to network theory, nodes and edges are the most essential factors. Since the combat network is an abstract based on military equipment and enemy targets, each element must be described as a node or edge of the combat network.

2.1. Combat Network Model. In collaborative combat systems, combat equipment with different functions, such as detection, communication, command, and attack, are closely linked and constitute a network through unidirectional edges. The systems include a collaborative sensing network, collaborative decision network, and collaborative attack network.

This paper regards the enemy targets and combat equipment that can independently accomplish combat activities as network nodes and regards the information transformation and combat activities as network edges.

2.2. Network Nodes. According to the combat network model $[8,9]$ proposed by Cares and the OODA cycle model [7], the nodes are divided into the sensors (manned reconnaissance aircraft $S_{1}$ and unmanned reconnaissance aircraft $S_{2}$ ), decision-makers (manned director aircraft $D_{1}$ and unmanned director aircraft $D_{2}$ ), influencers (manned fighter $I_{1}$ and unmanned fighter $I_{2}$ ), and the target $T$. The network nodes are shown in Figure 1.

$\varphi_{N_{\varphi}}$ is used to represent all of the nodes, $\varphi$ represents the type of the node, $\varphi \in\{T, S, D, I\}$, and $N_{\varphi}$ represents the number of $\varphi$ in the network. Different types of nodes have 

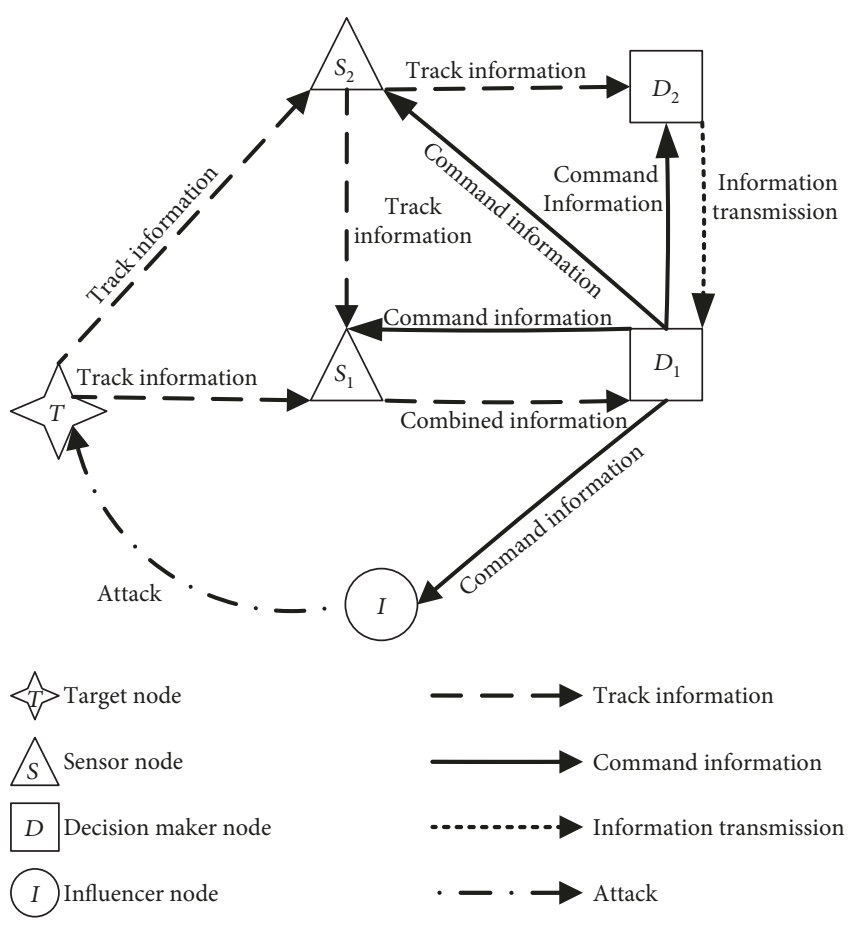

FIgURE 2: Network edges.

various technology indexes. The technology indexes for node $\varphi_{i}$ can be denoted as $x_{1}^{\varphi_{i}}, x_{2}^{\varphi_{i}}, \ldots, x_{n_{\varphi}}^{\varphi_{i}}$.

The target node $T$ and its properties are expressed as $x_{T}=\left(x_{1}^{T}, x_{2}^{T}, \cdots, x_{7}^{T}\right)$, where $x_{j}^{T}(j=1,2, \cdots, 7)$ is the camouflage coefficient, survivability coefficient, maneuvering velocity, warning time, anti-infrared coefficient, antiradar coefficient, and antioptical coefficient, respectively.

The sensor node $S$ and its properties are expressed as $x_{S}=\left(x_{1}^{S}, x_{2}^{S}, \cdots, x_{10}^{S}\right)$, where $x_{j}^{S}(j=1,2, \cdots, 10)$ is the maneuvering velocity, scan frequency, maximum detection range, detection precision, identification probability, communication coverage, transmission rate, communication quality, capacity, and delay, respectively.

The decision-maker node $D$ and its properties are expressed as $x_{D}=\left(x_{1}^{D}, x_{2}^{D}, \cdots, x_{8}^{D}\right)$, where $x_{j}^{D}(j=1,2, \cdots, 8)$ is the response time, throughput, precision, communication coverage, transmission rate, communication quality, capacity, and delay, respectively.

The influencer node $I$ and its properties are expressed as $x_{I}=\left(x_{1}^{I}, x_{2}^{I}, \cdots, x_{8}^{I}\right)$, where $x_{j}^{I}(j=1,2, \cdots, 8)$ is the maneuvering velocity, killing radius, firing accuracy, communication coverage, transmission rate, communication quality, capacity, and delay, respectively.

2.3. Network Edges. The edge model is based on the capability index of the weapon interaction, which is derived from the respective weapon performances. The environmental factor is ignored here.

A unidirectional edge between nodes represents the information exchange or combat tasks. According to the description of the main tasks, eight edges between the four nodes are generally considered, namely, $T-S, S-S, S-D$,
$D_{2}-D_{1}, D_{1}-D_{2}, D-S, D-I$, and $I-T$. The network edges are shown in Figure 2.

(1) $T$-S: the $T$-S edge mainly measures the reconnaissance ability of sensor node $S$, which can be measured from the following three aspects: finding, tracking, and recognition.

The finding ability $p_{f}$ is expressed as follows:

$$
p_{f}=1-\left(1-g_{i}\right)^{x_{2}^{S}}
$$

where $g_{i}$ represents the probability that $S$ can find $T$ in its $i$ th detection. If the search probability of sensor node $S p_{\text {search }}$ and the hiding probability of enemy target $T p_{\text {hide }}$ are considered, then

$$
\begin{gathered}
g_{i}=\frac{k \cdot p_{\text {search }}}{x_{1}^{T} \cdot p_{\text {hide }}}, \quad 0<k<1, \\
p_{\text {search }}=\exp \left(-\sum_{i=1}^{4} \omega_{x_{i}^{S}} \ln \left(\frac{1}{R_{x_{i}^{S}}}\right)\right), \\
p_{\text {hide }}=\exp \left(-\sum_{i=5}^{7} \omega_{x_{i}^{T}} \ln \left(\frac{1}{R_{x_{i}^{T}}}\right)\right),
\end{gathered}
$$

where $k$ is an environmental parameter (here, $k=0.9$ ), $R_{x_{i}^{\varphi}}$ represents the membership function of $x_{i}^{\varphi}$, and $\omega_{x_{i}^{\varphi}}$ represents the weight of each index.

Regarding the tracking ability $p_{t}$, if the tracking probability of sensor node $S p_{t r}$ and the antitrack probability of enemy target $T p_{z}$ are considered, then

$$
\begin{aligned}
& p_{t}=\frac{k_{z} \cdot p_{t r}}{x_{1}^{T} \cdot p_{z}}, \quad 0<k_{z}<1, \\
& p_{t r}=\exp \left(-\sum_{i=1}^{3} \omega_{x_{i}^{S}} \ln \left(\frac{1}{R_{x_{i}^{S}}}\right)\right), \\
& p_{z}=\exp \left(-\omega_{x_{3}^{T}} \ln \left(\frac{1}{R_{x_{3}^{T}}}\right)-\omega_{x_{4}^{T}} \ln \left(\frac{1}{R_{x_{4}^{T}}}\right)\right),
\end{aligned}
$$

where $k_{z}$ is an environmental parameter (here, $k=0.9$ ).

The recognition ability $p_{r}$ is equal to the membership function $R_{x_{5}^{s}}$.

$E_{T-S}$ represents the reconnaissance ability of $T-S$ and is expressed as follows:

$$
E_{T-S}=\exp \left(-\sum_{i \in\{f, t, r\}} \omega_{i} \ln \left(\frac{1}{p_{i}}\right)\right),
$$

where $\omega_{i}(i \in\{f, t, r\})$ represents the weights of finding, tracking, and recognition. 
(2) $S$-S: the $S$-S edge indicates that unmanned reconnaissance aircraft $S_{2}$ sends information $\left(\right.$ Inform $\left._{2}\right)$ to manned reconnaissance aircraft $S_{1}$. On the one side, $S_{1}$ searches for enemy target information ( Inform $_{1}$ ), and on the other side, Inform ${ }_{2}$ is combined with Inform $_{1}$ to obtain other important information. These two steps embody the emergence and cooperativity of the combat systems. The $S$-S edge mainly measures the information transmission ability of sensor node $S$. $p_{i}(i=1,2, \cdots, 5)$ represents the communication coverage, transmission rate, communication quality, capacity, and delay, respectively, as follows:

$$
\begin{aligned}
& p_{1}=\left\{\begin{array}{l}
1, \quad d_{S_{1}-S_{2}} \leq \min \left\{x_{6}^{S_{1}}, x_{6}^{S_{2}}\right\}, \\
0, \quad d_{S_{1}-S_{2}}>\min \left\{x_{6}^{S_{1}}, x_{6}^{S_{2}}\right\},
\end{array}\right. \\
& p_{2}=\min \left\{x_{7}^{S_{1}}, x_{7}^{S_{2}}\right\}, \\
& p_{3}=x_{8}^{S_{1}} \cdot x_{8}^{S_{2}}, \\
& p_{4}=\min \left\{x_{9}^{S_{1}}, x_{9}^{S_{2}}\right\}, \\
& p_{5}=x_{10}^{S_{1}}+x_{10}^{S_{2}} .
\end{aligned}
$$

$E_{S-S}$ represents the information transmission ability of $S-S$ and is expressed as follows:

$$
E_{S-S}=p_{1}{ }^{*} \exp \left(-\sum_{i=2}^{5} \omega_{i} \ln \left(\frac{1}{R_{i}}\right)\right)
$$

where $R_{i}$ represents the membership function of $p_{i}(i=1,2, \cdots, 5)$.

(3) $S$ - $D$ : the $S-D$ edge indicates that information collected by sensor node $S$ is transmitted to decisionmaker node $D$. According to the characteristics of $S-D$, the information transmission ability between these nodes is mainly considered. The information transmission ability of $S-D$ is shown as follows:

$$
E_{S-D}=p_{1}{ }^{*} \exp \left(-\sum_{i=2}^{5} \omega_{i} \ln \left(\frac{1}{R_{i}}\right)\right)
$$

(4) $D_{2}-D_{1}$ : the $D_{2}-D_{1}$ edge indicates that information analyzed by unmanned director aircraft $D_{2}$ is transmitted to manned director aircraft $D_{1}$. When determining the information sharing ability of $D_{2}-D_{1}$, the information transmission ability $E_{1}$ and information processing ability $E_{2}$ must be considered. The information transmission ability of $D_{2}-D_{1}$ is shown as follows:

$$
E_{1}=p_{1}{ }^{*} \exp \left(-\sum_{i=2}^{5} \omega_{i} \ln \left(\frac{1}{R_{i}}\right)\right) .
$$

$E_{2}$ represents the information processing ability of $D_{2}$ and is expressed as follows:

$$
E_{2}=\exp \left(-\sum_{i=1}^{3} \omega_{i} \ln \left(\frac{1}{R_{i}}\right)\right)
$$

where $R_{i}$ represents the membership function of $x_{i}^{D_{2}}(i=1,2,3)$.

$E_{D_{2}-D_{1}}$ represents the information sharing ability of $D_{2}-D_{1}$ and is expressed as follows:

$$
E_{D_{2}-D_{1}}=\sqrt{E_{1} \cdot E_{2}} \text {. }
$$

(5) $D_{1}-D_{2}$ : the $D_{1}-D_{2}$ edge indicates that manned director aircraft $D_{1}$ conveys commands to unmanned director aircraft $D_{2}$. When determining the command and control ability of $D_{1}-D_{2}$, the information transmission ability of $D_{1}-D_{2}$ and information processing ability of $D_{1}$ must be considered. $E_{D_{1}-D_{2}}$ represents the command and control ability of $D_{1}-D_{2}$ and can be expressed as follows:

$$
E_{D_{1}-D_{2}}=\sqrt{E_{1} \cdot E_{2}} \text {. }
$$

(6) $D$-S: the $D$-S edge indicates that decision-maker node $D$ conveys commands to sensor node $S$ such that $S$ can further detect, track, and identify the enemy targets to obtain more specific information. When determining the command and control ability of $D-S$, the information transmission ability of $D-S$ and information processing ability of $D$ must be considered. $E_{D-S}$ represents the command and control ability of $D-S$ and can be expressed as follows:

$$
E_{D-S}=\sqrt{E_{1} \cdot E_{2}}
$$

(7) $D-I$ : the $D-I$ edge indicates that decision-maker node $D$ conveys attack commands to influencer node $I$. The $D-I$ edge depends on the information transmission ability, which is shown as follows:

$$
E_{D-I}=p_{1}{ }^{*} \exp \left(-\sum_{i=2}^{5} \omega_{i} \ln \left(\frac{1}{R_{i}}\right)\right)
$$

(8) $I$ - $T$ : the $I-T$ edge mainly measures the attack ability of influencer node $I$, which can be measured by hit probability $p_{h}$ and damage probability $p_{d} \cdot p_{h}$ is defined as follows: 


$$
p_{h}= \begin{cases}0, & x_{1}^{I} \cdot t_{I-T}+x_{2}^{I}<d_{I-T}+x_{3}^{T} \cdot x_{4}^{T}, \\ 1, & x_{1}^{I} \cdot t_{I-T}+x_{2}^{I} \geq d_{I-T}+x_{3}^{T} \cdot x_{4}^{T} .\end{cases}
$$

For the damage probability $p_{d}$, if the damage ability of influencer node $I p_{a}$ and the survival ability of enemy target $T p_{o}$ are considered, then

$$
\begin{aligned}
& p_{d}=\frac{k_{a}^{*} p_{a}}{p_{o}}, \quad 0<k_{a}<1, \\
& p_{a}=\exp \left(-\sum_{i=1}^{3} \omega_{x_{i}^{I}} \ln \left(\frac{1}{R_{x_{i}^{I}}}\right)\right),
\end{aligned}
$$

where $k_{a}$ is an environmental parameter (here, $k_{a}=0.85$ ).

The survival ability $p_{o}$ is equal to the membership function $R_{x_{2}^{T}}$.

$E_{I-T}$ represents the attack ability of $I-T$ and can be expressed as follows:

$$
E_{I-T}=\sqrt{p_{h} \cdot p_{d}}
$$

\section{Evaluation of MAV/UAV Collaborative Combat}

3.1. Evaluation Method. Based on the definition of the nodes and edges, the operation loop is used to integrate combat activities, such as detection, command, and attack. An operation loop is a closed network comprising of a series of nodes and unidirectional edges connected by material, energy, or information. For a given enemy target $T$, our combat entities can build an operation loop OL. The impact of a series of combat activities on $T$ is the capability of the operation loop $O L$.

The improved information entropy is used to measure the combat activities, and the operation loop capability is evaluated by this information. Next, the uncertainty contained in the combat activities of each edge is analyzed, and the formulas for improved information entropy are given.

(1) $T$-S: the stronger reconnaissance capability $E_{T-S}$ of the $T$-S edge means that the information uncertainty of the target is lower and that the collaborative combat capability is stronger when more accurate information on the enemy targets is obtained by $S . E_{T-S}$ represents the reconnaissance capability of $T-S$; therefore, the self-information entropy of $T-S$ is expressed as follows:

$$
S_{T-S}=-\log E_{T-S} .
$$

(2) $S$-S: the $S$-S edge implies a synergic relationship between the $S$ nodes, which refers to the relationship between $S_{2}-S_{1}$ and $T-S_{1}$. This edge mainly reflects the information fusion ability of $S_{1}$. The information entropy after synergy can be represented by the joint entropy method.

$$
H(X, Y)=H(X)+H(Y)-I(X ; Y) .
$$

$X$ and $Y$ represent the operational activities of $S_{2}-S_{1}$ and $T-S_{1}$, respectively.

$$
\begin{aligned}
H(X) & =E_{S_{2}-S_{1}}, \\
H(Y) & =E_{T-S_{1}}, \\
H(X, Y) & =E_{S-S}, \\
I(X ; Y) & =-\sum_{y} \sum_{x} p(x, y) \log \frac{p(x, y)}{p(x) p(y)} \\
& =-\sum_{y} \sum_{x} p(y \mid x) p(x) \log \frac{p(y \mid x)}{p(y)} .
\end{aligned}
$$

According to the definition of mutual information in equation (26), the impact that the information obtained and transmitted by $S_{2}$ to the reconnaissance ability of $S_{1}$ is measured by $p(y \mid x)$. $p(x)$ and $p(y)$ represent the completion degree of $S_{2}-S_{1}$ and $T-S_{1}$, respectively, which are equal to $E_{S_{2}-S_{1}}$ and $E_{T-S_{1}}$. Then, $p(y \mid x)$ is defined as follows:

$$
p(y \mid x)=k_{s} \cdot \frac{E_{T-S_{1}}}{1-E_{S_{2}-S_{1}}},
$$

where $k_{s}$ is an influence coefficient (here, $k_{s}=0.2$ ). $S_{1}$ is more likely to obtain more information through fusion when the information transmitted by $S_{2}-S_{1}$ is more accurate.

$$
I(X ; Y)=-k_{s} \cdot \frac{E_{T-S_{1}}}{1-E_{S_{2}-S_{1}}} \cdot E_{S_{2}-S_{1}} \cdot \log \left(\frac{k_{s}}{1-E_{S_{2}-S_{1}}}\right)
$$

Therefore, the self-information entropy of $S-S$ is expressed as follows:

$$
S_{S-S}=-\log \left(E_{S-S}\right)=-\log (H(X, Y)) .
$$

(3) $S$ - $D$ : the information uncertainty of $S-D$ mainly originates from communication. Thus, the selfinformation entropy of $S-S$ combat activities is expressed as follows:

$$
S_{S-D}=-\log E_{S-D}
$$

(4) $D_{2}-D_{1}$ : unmanned director aircraft $D_{2}$ analyzes the information of the enemy and sends it to manned director aircraft $D_{1}$. The uncertainty inherent in this process mainly lies in the information transmission ability and information processing ability.

$$
S_{D_{2}-D_{1}}=-\log E_{D_{2}-D_{1}}=-\log \sqrt{E_{1} \cdot E_{2}} .
$$


(5) $D_{1}-D_{2}$ : considering the current situation, manned director aircraft $D_{1}$ further determines the targets and provides appropriate measures. Similarly, the uncertainty inherent in this process mainly lies in the information transmission ability and information processing ability.

$$
S_{D_{1}-D_{2}}=-\log E_{D_{1}-D_{2}}=-\log \sqrt{E_{1} \cdot E_{2}} .
$$

(6) $D$-S: the $D-S$ edge describes the process of director aircraft $D$ communicating surveillance commands to sensor node $S$, which is based on the data analysis results. Similarly, the uncertainty inherent in this process mainly lies in the information transmission ability and information processing ability.

$$
S_{D-S}=-\log E_{D-S}=-\log \sqrt{E_{1} \cdot E_{2}} .
$$

(7) $D-I$ : the $D-I$ edge describes the process of director aircraft $D$ communicating attack commands to influencer node $I$. The uncertainty inherent in this process mainly lies in the information transmission ability. Therefore, the self-information entropy of $D-I$ can be expressed as follows:

$$
S_{D-I}=-\log E_{D-I}
$$

(8) $I-T$ : the $I-T$ edge describes the process of influencer node $I$ attacking enemy target $T$. The uncertainty inherent in this process mainly lies in the hit probability $p_{h}$ and damage probability. Therefore, the self-information entropy of $I-T$ is expressed as shown as follows:

$$
S_{I-T}=-\log E_{I-T}=-\log \sqrt{p_{h} \cdot p_{d}} .
$$

At this point, the description of the self-information entropy of each edge has been completed. Next, the length of each edge, that is, the distance between two adjacent nodes, is defined.

Definition 1. The length of $e_{i j}$. The length of the unidirectional edge $e_{i j}$ between nodes $x_{i}$ and $x_{j}$ is expressed as $l_{i j}=S_{i-j}$.

Definition 2. The distance from $x_{i}$ to $x_{j}$. If there are some intermediate nodes $x_{i}, x_{j} \notin\left\{x_{1}, x_{2}, \cdots, x_{k}\right\}, i \neq j$ and a set of unidirectional edges $E_{i j}=\left\{x_{i} x_{1}, x_{1} x_{2}, \cdots x_{k-1} x_{k}, x_{k} x_{j}\right\}$ between $x_{i}$ and $x_{j}$, then a path $r_{i j}$ exists from $x_{i}$ to $x_{j}$. The distance $d_{i j}$ is related to the length of path $r_{i j}$, which can be described as follows:

$$
d_{i j}=\frac{N-1}{N} \sqrt[N]{\prod_{e_{p q} \in E_{i j}} l_{p q}}=\frac{N-1}{N} \sqrt[N]{\prod_{e_{p q} \in E_{i j}} S_{p-q}},
$$

where $N=k+1$

According to the distance $d_{i j}$, the distance between the two nodes in the combat network is equal to the total uncertainties of all combat activities. For a given enemy target $T_{m}$, the length of operation loop $O L_{m_{n}}$ is described as follows:

$$
d_{O L_{m_{n}}}=\frac{N-1}{N} \sqrt[N]{\prod_{e_{p q} \in E_{m_{n}}} l_{p q}}=\frac{N-1}{N} \sqrt[N]{\prod_{e_{p q} \in E_{m_{n}}} S_{p-q}},
$$

where $N$ is the number of edges in the operation loop and $E_{m_{n}}$ is the set of all unidirectional edges.

The length of the operation loop can be used to measure the combat capability to the given target. The length is proportional to the information uncertainty and inversely proportional to the combat capability. The operation loop is expressed as follows:

$$
O L C_{m_{n}}=\exp \left(-d_{O L_{m_{n}}}\right)
$$

For different military actions against $T_{m}$, the combat capability is the comprehensive uncertainty of all operation loops. Suppose that $M$ military actions are formulated for target $M$, that is, $M$ operation loops are made. Then, the comprehensive uncertainty of all operation loops is described as follows:

$$
d_{O L_{m}}=\frac{1}{\sum_{j=1}^{M} 1 / d_{O L_{m_{j}}}} .
$$

The combat capability AOC for target $T_{m}$ is calculated as follows:

$$
\mathrm{AOC}=\exp \left(-\frac{1}{\sum_{j=1}^{M} 1 / d_{O L_{m_{j}}}}\right)
$$

Based on the weighted calculation, the evaluation indexes of the MAV/UAV collaborative combat capability can be obtained as follows:

$$
Y=\sum_{i=1}^{N_{T}} \omega_{i} \cdot \mathrm{AOC}_{i}
$$

3.2. Evaluation Procedure. Based on the above evaluation method, the evaluation procedure of the MAV/UAV collaborative combat capability can be summarized as follows:

Step 1: Analyze the strategic missions and define the set of enemy targets $T=\left\{T_{1}, T_{1}, \cdots T_{N_{T}}\right\}$ 


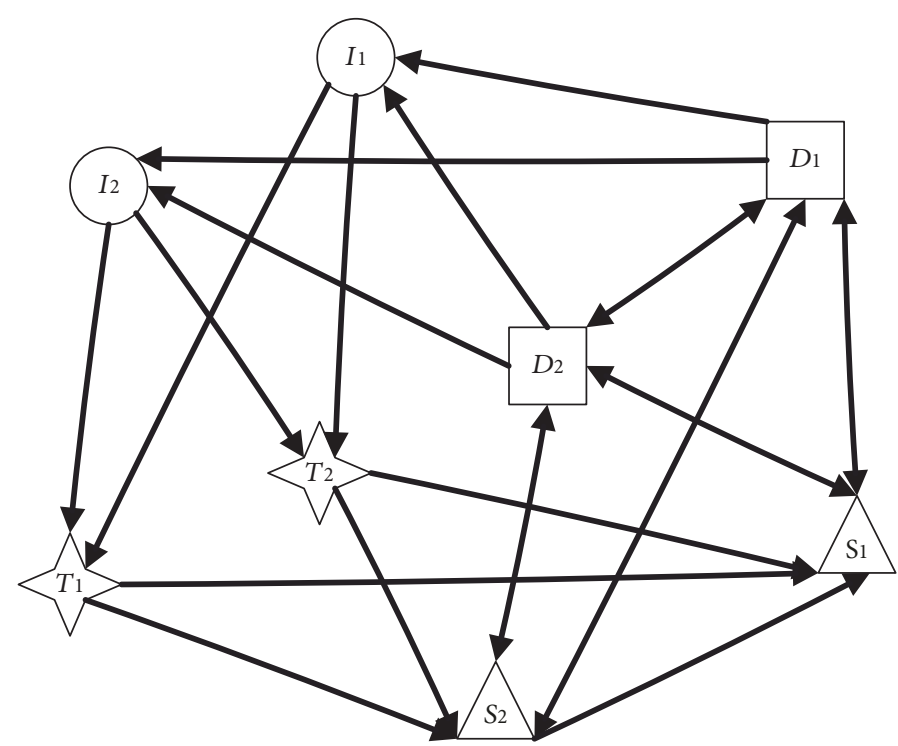

FIgURE 3: An MAV/UAV collaborative combat network.

TABLe 1: Tactical indexes of $T$.

\begin{tabular}{lccc}
\hline Name & Code & $T_{1}$ & $T_{2}$ \\
\hline Camouflage coefficient & $\mathrm{x} 1$ & 3 & 3 \\
Survivability coefficient $(\mathrm{mm})$ & $\mathrm{x} 2$ & 800 & 500 \\
Maneuvering velocity $(\mathrm{km} / \mathrm{h})$ & $\mathrm{x} 3$ & 70 & 50 \\
Warning time $(\mathrm{s})$ & $\mathrm{x} 4$ & 0.6 & 0.5 \\
Anti-infrared coefficient & $\mathrm{x} 5$ & 8 & 7 \\
Antiradar coefficient & $\mathrm{x} 6$ & 6 & 5 \\
Antioptical coefficient & $\mathrm{x} 7$ & 7 & 7 \\
\hline
\end{tabular}

Step 2: According to the targets, determine the combat entities and establish the model of nodes and edges

Step 3: Identify the operation loops and calculate their combat capabilities

Step 4: Calculate the combat capability of all operational loops for each target

Step 5: Obtain the MAV/UAV collaborative combat capability by calculating the combat capabilities of the operation loops aimed at all the targets

\section{Example}

The network framework provided in this example is an ideal model. To simplify the model and highlight the process of the cooperative combat capability evaluation, we considered the simplest one. In the example, only eight nodes are considered, that is, each node represents an UAV or an MAV with different functions. In practical applications, nodes can be added based on the specific combat environment, and the edges can be determined by the specific combat assignments.
Some of the parameter assignments in this example come from experience summarization. After much investigation and calculation of statistics, the most representative parameters are selected from the functional parameters of reconnaissance aircrafts and combat aircrafts, such as the maneuvering velocity, scan frequency, and maximum detection range of the sensor node $S$. The other part is imaginary scene representation, such as the anti-infrared coefficient, antiradar coefficient, and antioptical coefficient of the target node $T$. The parameters selected in this example are just used as examples. In practical applications, the correct parameters for the actual model can be substituted.

4.1. Nodes of the Network. Based on the above modeling process, the MAV/UAV collaborative combat network shown in Figure 3 can be obtained.

The technical indexes of each node are assigned according to the description of the nodes in the collaborative combat network. In calculating the information entropy and combat capability of each edge, the membership functions are determined in combination with actual combat and expert experience.

(1) Target node $T$ : the hypothetical target nodes and their technical indexes in this example are shown in Table 1.

The membership function of the survivability coefficient is shown as follows:

$$
R_{x_{2}^{T}}= \begin{cases}1, & 800 \leq x_{2}^{T} \\ 0.7, & 500 \leq x_{2}^{T}<800 \\ 0.3, & 300 \leq x_{2}^{T}<500 \\ 0.1, & x_{2}^{T}<300\end{cases}
$$


TABLE 2: Tactical indexes of $S$.

\begin{tabular}{lccc}
\hline Name & Code & $S_{1}$ & $S_{2}$ \\
\hline Maneuvering velocity $(\mathrm{km} / \mathrm{h})$ & $\mathrm{x} 1$ & 1000 & 700 \\
Scan frequency $(\mathrm{Hz})$ & $\mathrm{x} 2$ & 7 & 8 \\
Maximum detection range $(\mathrm{km})$ & $\mathrm{x} 3$ & 3500 & 2500 \\
Detection precision $\left(\mathrm{m}^{2}\right)$ & $\mathrm{x} 4$ & 5 & 6 \\
Identification probability & $\mathrm{x} 5$ & 0.65 & 0.85 \\
Communication coverage $(\mathrm{km})$ & $\mathrm{x} 6$ & 3000 & 2500 \\
Transmission rate $(\mathrm{bps})$ & $\mathrm{x} 7$ & 15 & 12 \\
Communication quality & $\mathrm{x} 8$ & 0.2 & 0.3 \\
Communication capacity $(\mathrm{MHz} / \mathrm{s})$ & $\mathrm{x} 9$ & 40 & 30 \\
Communication delay $(\mathrm{ms})$ & $\mathrm{x} 10$ & 5.5 & 4 \\
\hline
\end{tabular}

The weights of the maneuvering velocity and warning time are $\omega_{x_{3}^{T}}=0.3$ and $\omega_{x_{4}^{T}}=0.7$, and their membership functions are shown, respectively, as follows:

$$
\begin{gathered}
R_{x_{3}^{T}}= \begin{cases}1, & 70 \leq x_{3}^{T}, \\
0.5, & 50 \leq x_{3}^{T}<70, \\
0.3, & 30 \leq x_{3}^{T}<50, \\
0.1, & x_{3}^{T}<30,\end{cases} \\
R_{x_{4}^{T}}= \begin{cases}1, & 0.7 \leq x_{4}^{T}, \\
0.7, & 0.5 \leq x_{4}^{T}<0.7, \\
0.3, & 0.3 \leq x_{4}^{T}<0.5, \\
0.1, & x_{4}^{T}<0.3 .\end{cases}
\end{gathered}
$$

The anti-infrared, antiradar, and antioptical weights are $\omega_{x_{5}^{T}}=0.25, \omega_{x_{6}^{T}}=0.6$, and $\omega_{x_{7}^{T}}=0.15$, and their membership functions are shown, respectively, as follows:

$$
\begin{aligned}
& R_{x_{5}^{T}}= \begin{cases}1, & 6<x_{5}^{T}, \\
0.6, & 4<x_{5}^{T} \leq 6, \\
0.2, & x_{5}^{T} \leq 4,\end{cases} \\
& R_{x_{6}^{T}}= \begin{cases}1, & 5<x_{6}^{T}, \\
0.5, & 2<x_{6}^{T} \leq 5, \\
0.2, & x_{6}^{T} \leq 2,\end{cases} \\
& R_{x_{7}^{T}}= \begin{cases}1, & 10 \leq x_{7}^{T}, \\
0.6, & 5 \leq x_{7}^{T}<10, \\
0.1, & x_{7}^{T}<5 .\end{cases}
\end{aligned}
$$

When calculating the reconnaissance capability of $T-S$, the weights of finding, tracking, and identification are each equal to $1 / 3$.

(2) Sensor node $S$ : the technical indexes of the $S$ nodes mentioned above are shown in Table 2.
TABLe 3: Tactical indexes of $D$.

\begin{tabular}{lccc}
\hline Name & Code & $D_{1}$ & $D_{2}$ \\
\hline Response time (s) & $\mathrm{x} 1$ & 0.02 & 0.04 \\
Throughput (bps) & $\mathrm{x} 2$ & 8 & 4 \\
Precision & $\mathrm{x} 3$ & 0.9 & 0.6 \\
Communication coverage (km) & $\mathrm{x} 4$ & 2600 & 2000 \\
Transmission rate (bps) & $\mathrm{x} 5$ & 8 & 5 \\
Communication quality & $\mathrm{x} 6$ & 0.1 & 0.12 \\
Communication capacity (MHz/s) & $\mathrm{x} 7$ & 60 & 30 \\
Communication delay (ms) & $\mathrm{x} 8$ & 4 & 4 \\
\hline
\end{tabular}

The maneuvering velocity, scan frequency, maximum detection range, and detection precision weights are $\omega_{x_{1}^{s}}=$ $0.2, \omega_{x_{2}^{s}}=0.3, \omega_{x_{3}^{s}}=0.15$, and $\omega_{x_{4}^{s}}=0.35$, respectively, and their membership functions are shown, respectively, as follows:

$$
\begin{aligned}
& R_{x_{1}^{S}}= \begin{cases}1, & 900 \leq x_{1}^{S}, \\
0.8, & 700 \leq x_{1}^{S}<900, \\
0.5, & 500 \leq x_{1}^{S}<700, \\
0.2, & x_{1}^{S}<500,\end{cases} \\
& R_{x_{2}^{S}}= \begin{cases}1, & 6 \leq x_{2}^{S}, \\
0.8, & 5 \leq x_{2}^{S}<6, \\
0.5, & 3 \leq x_{2}^{S}<5, \\
0.2, & x_{2}^{S}<3,\end{cases} \\
& R_{x_{3}^{S}}= \begin{cases}1, & 3000 \leq x_{3}^{S}, \\
0.8, & 2000 \leq x_{3}^{S}<3000, \\
0.3, & x_{3}^{S}<2000,\end{cases} \\
& R_{x_{4}^{S}}= \begin{cases}0.2, & 7 \leq x_{4}^{S}, \\
0.5, & 5 \leq x_{4}^{S}<7, \\
0.8, & 4 \leq x_{4}^{S}<5, \\
1, & x_{4}^{S}<4,\end{cases} \\
& R_{x_{5}^{S}}= \begin{cases}1, & 0.9 \leq x_{5}^{S}, \\
0.8, & 0.8 \leq x_{5}^{S}<0.9, \\
0.4, & 0.6 \leq x_{5}^{S}<0.8, \\
0.2, & x_{5}^{S}<0.6 .\end{cases}
\end{aligned}
$$

(3) Decision-maker node $D$ : the technical indexes of the decision-maker nodes in our collaborative combat systems are shown in Table 3.

When considering the information processing capability of $D$, the following three tactical indexes exist: response time, 
TABLe 4: Tactical indexes of $I$.

\begin{tabular}{lccc}
\hline Name & Code & $I_{1}$ & $I_{2}$ \\
\hline Maneuvering velocity $(\mathrm{km} / \mathrm{h})$ & $\mathrm{x} 1$ & 1600 & 2000 \\
Killing radius $(\mathrm{km})$ & $\mathrm{x} 2$ & 20 & 30 \\
Firing accuracy $(\mathrm{m})$ & $\mathrm{x} 3$ & 3 & 5 \\
Communication coverage $(\mathrm{km})$ & $\mathrm{x} 4$ & 2000 & 2000 \\
Transmission rate $(\mathrm{bps})$ & $\mathrm{x} 5$ & 8 & 7.5 \\
Communication quality & $\mathrm{x} 6$ & 0.1 & 0.12 \\
Communication capacity $(\mathrm{MHz} / \mathrm{s})$ & $\mathrm{x} 7$ & 50 & 25 \\
Communication delay $(\mathrm{ms})$ & $\mathrm{x} 8$ & 3 & 2 \\
\hline
\end{tabular}

throughput, and precision; their weights are $\omega_{x_{1}^{D}}=0.3$, $\omega_{x_{2}^{D}}=0.25$, and $\omega_{x_{3}^{D}}=0.45$, respectively, and their membership functions are shown, respectively, as follows:

$$
\begin{aligned}
& R_{x_{1}^{D}}= \begin{cases}0.2, & 0.06 \leq x_{1}^{D}, \\
0.5, & 0.03 \leq x_{1}^{D}<0.06, \\
0.8, & 0.01 \leq x_{1}^{D}<0.03, \\
1, & x_{1}^{D}<0.01,\end{cases} \\
& R_{x_{2}^{D}}= \begin{cases}1, & 8 \leq x_{1}^{D}, \\
0.7, & 6 \leq x_{1}^{D}<8, \\
0.3, & 4 \leq x_{1}^{D}<6, \\
0.1, & x_{1}^{D}<4,\end{cases} \\
& R_{x_{3}^{D}}= \begin{cases}1, & 0.9 \leq x_{3}^{D}, \\
0.7, & 0.7 \leq x_{3}^{D}<0.9, \\
0.5, & 0.4 \leq x_{3}^{D}<0.7, \\
0.2, & x_{3}^{D}<0.4 .\end{cases}
\end{aligned}
$$

(4) Influencer node $I$ : the technical indexes of $I$ are shown in Table 4.

When considering the attack ability of $I$, the following three tactical indexes exist: maneuvering velocity, killing radius, and firing accuracy; their weights are $\omega_{x_{1}^{I}}=0.3$, $\omega_{x_{2}^{I}}=0.3$, and $\omega_{x_{3}^{I}}=0.4$, respectively. Their membership functions are shown, respectively, as follows:

$$
R_{x_{1}^{I}}= \begin{cases}1, & 2000 \leq x_{1}^{I}, \\ 0.8, & 1500 \leq x_{1}^{I}<2000, \\ 0.5, & 1000 \leq x_{1}^{I}<1500, \\ 0.25, & x_{1}^{I}<1000\end{cases}
$$

$$
\begin{aligned}
& R_{x_{2}^{I}}= \begin{cases}1, & 30 \leq x_{2}^{I}, \\
0.8, & 20 \leq x_{2}^{I}<30, \\
0.5, & 10 \leq x_{2}^{I}<20, \\
0.25, & x_{2}^{I}<10,\end{cases} \\
& R_{x_{3}^{I}}= \begin{cases}0.1, & 6 \leq x_{3}^{I}, \\
0.5, & 4 \leq x_{3}^{I}<6, \\
0.8, & 2 \leq x_{3}^{I}<4, \\
1, & x_{3}^{I}<2 .\end{cases}
\end{aligned}
$$

In addition, when considering the information transmission capacity between nodes, the following four tactical indexes are considered: transmission rate, communication quality, capacity, and delay, with weights of $\omega_{3}=0.3, \omega_{4}=$ 0.2 , and $\omega_{5}=0.2$, respectively. Their membership functions are shown, respectively, as follows:

$$
\begin{aligned}
& R_{2}= \begin{cases}1, & 15 \leq p_{2}, \\
0.8, & 10 \leq p_{2}<15, \\
0.6, & 5 \leq p_{2}<10, \\
0.2, & p_{2}<5,\end{cases} \\
& R_{3}= \begin{cases}0.2, & 0.3 \leq p_{3}, \\
0.6, & 0.2 \leq p_{3}<0.3, \\
0.8, & 0.1 \leq p_{3}<0.2, \\
1, & p_{3}<0.1,\end{cases} \\
& R_{4}= \begin{cases}1, & 60 \leq p_{4}, \\
0.8, & 50 \leq p_{4}<60, \\
0.6, & 40 \leq p_{4}<50, \\
0.2, & p_{4}<40,\end{cases} \\
& R_{5}= \begin{cases}0.25, & 4 \leq p_{5}, \\
0.6, & 3 \leq p_{5}<4, \\
0.8, & 2 \leq p_{5}<3, \\
1, & p_{5}<2 .\end{cases}
\end{aligned}
$$

4.2. Network Edges. According to the technical indexes of the different nodes in the MAV/UAV collaborative combat network, the capability and information entropy of each edge can be calculated when combined with the analysis of the relationships among the nodes. The capability of each edge can be obtained by substituting the technical indexes of the different nodes into equations (4)-(20). Then, the information entropy of each edge can be obtained by substituting the capabilities of the different edges into equations (21)(35), as shown in Table 5. 
TABLE 5: Edge capability and length.

\begin{tabular}{lccccccc}
\hline Number & Edge & Capability & Length & Number & Edge & Capability & Length \\
\hline 1 & $T_{1}-S_{1}$ & 0.5120 & 0.6694 & 13 & $D_{1}-S_{1}$ & 0.7131 & 0.3382 \\
2 & $T_{1}-S_{2}$ & 0.6312 & 0.4601 & 14 & $D_{1}-S_{2}$ & 0.6216 & 0.4755 \\
3 & $T_{2}-S_{1}$ & 0.5681 & 0.5655 & 15 & $D_{2}-S_{1}$ & 0.4189 & 0.8700 \\
4 & $T_{2}-S_{2}$ & 0.6982 & 0.3592 & 16 & $D_{2}-S_{2}$ & 0.4189 & 0.8700 \\
5 & $T_{1}-S_{2}-S_{1}$ & 0.8750 & 0.1335 & 17 & $D_{1}-I_{1}$ & 0.5886 & 0.5301 \\
6 & $T_{2}-S_{2}-S_{1}$ & 0.8658 & 0.1441 & 18 & $D_{1}-I_{2}$ & 0.4162 & 0.8766 \\
7 & $S_{1}-D_{1}$ & 0.5477 & 0.6020 & 19 & $D_{2}-I_{1}$ & 0.4162 & 0.8766 \\
8 & $S_{1}-D_{2}$ & 0.4162 & 0.8766 & 20 & $D_{2}-I_{2}$ & 0.4162 & 0.8766 \\
9 & $S_{2}-D_{1}$ & 0.4162 & 0.8766 & 21 & $I_{1}-T_{1}$ & 0.8246 & 0.1928 \\
10 & $S_{2}-D_{2}$ & 0.4162 & 0.8766 & 22 & $I_{1}-T_{2}$ & 0.9856 & 0.0145 \\
11 & $D_{1}-D_{2}$ & 0.6216 & 0.4755 & 23 & $I_{2}-T_{1}$ & 0.8026 & 0.2199 \\
12 & $D_{2}-D_{1}$ & 0.4189 & 0.8700 & 24 & $I_{2}-T_{2}$ & 0.9593 & 0.0416 \\
\hline
\end{tabular}

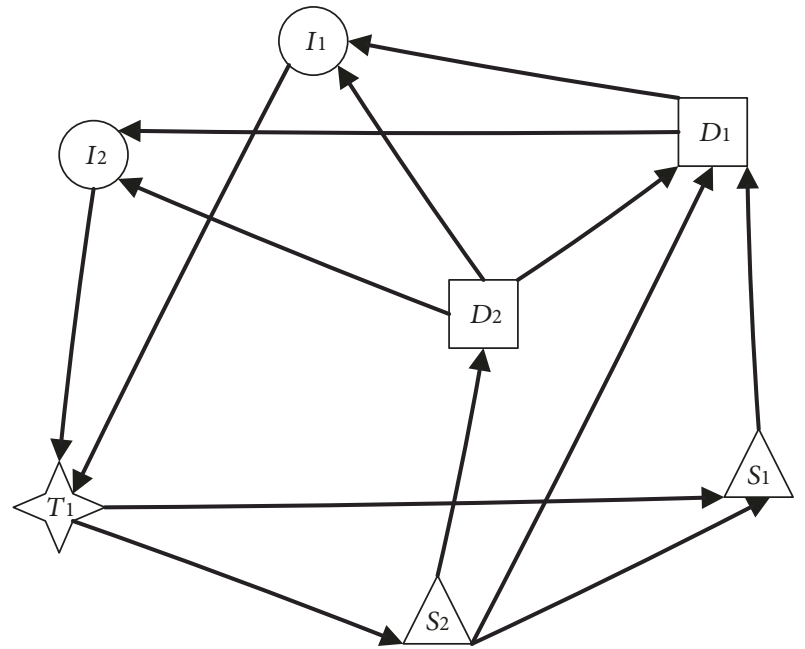

Figure 4: MAV/UAV collaborative combat network aimed at target $T_{1}$.

4.3. Capability Evaluation. The MAV/UAV collaborative combat network aimed at target $T_{1}$ can be formed by the military activities for target $T_{1}$, as shown in Figure 4 .

Referring to Table 5, the information entropy of the operation loops can be obtained by substituting the lengths of the corresponding edges into equation (37). According to equation (38), the capability of the operation loop can be obtained.

Table 6 shows the operation loops and the capability and length results aimed at target $T_{1}$.

Similarly, the MAV/UAV collaborative combat network aimed at target $T_{2}$ can be formed by the military activities for target $T_{2}$, as shown in Figure 5.

Table 7 shows the operation loops and the capability and length results aimed at target $T_{2}$.

According to equation (39), the comprehensive uncertainties of the operation loops aimed at targets $T_{1}$ and $T_{2}$ can be obtained, respectively. If these uncertainties are introduced in equation (40), the combat capability AOC for targets $T_{1}$ and $T_{2}$ can be obtained.
The combat capabilities synthesized by the operation loops aimed at targets $T_{1}$ and $T_{2}$ are calculated, respectively, as follows:

$$
\begin{aligned}
\mathrm{AOC}_{T_{1}} & =\exp \left(-d_{O L_{1}}\right) \\
& =\exp \left(-\frac{1}{\sum_{j=1}^{M} 1 / d_{O L_{m_{j}}}}\right) \\
& =\exp (-0.0226)=0.9777 \\
\mathrm{AOC}_{T_{2}} & =\exp \left(-d_{O L_{2}}\right) \\
& =\exp \left(-\frac{1}{\sum_{j=1}^{M} 1 / d_{O L_{m_{j}}}}\right) \\
& =\exp (-0.0210)=0.9792 .
\end{aligned}
$$

After analyzing the target weights, the overall combat capability can be determined. In this case, the weights of the two targets are $\omega_{T_{1}}=0.7$ and $\omega_{T_{2}}=0.3$; thus, the MAV/UAV collaborative combat capability is calculated as follows:

$$
\begin{aligned}
Y & =\sum_{i=1}^{2} \omega_{T_{i}} \cdot \mathrm{AOC}_{T_{i}} \\
& =0.7 \times 0.9777+0.3 \times 0.9792=0.9781 .
\end{aligned}
$$

\subsection{Analysis of the Result}

(1) Influence of the node in the operation loop on the combat capability: according to Table 5, when considering edges 1 to 4 , the reconnaissance and detection capabilities of unmanned reconnaissance aircraft $S_{2}$ are superior to those of $S_{1}$; when considering edges 11 to 12 and 11 to 16 , the command and detection capabilities of manned command aircraft $D_{1}$ are superior to those of $D_{2}$; and when considering edges 21 to 24 , the fire strike capability of manned 
TABLE 6: Capability and length of operation loops aimed at target $T_{1}$.

\begin{tabular}{lcccc}
\hline Number & Operation loop & Number of nodes & Length of operation loop & Capability of operation loop \\
\hline 1 & $T_{1}-S_{2}-S_{1}-D_{1}-I_{1}-T_{1}$ & 5 & 0.2503 & 0.7786 \\
2 & $T_{1}-S_{2}-S_{1}-D_{1}-I_{2}-T_{1}$ & 5 & 0.2817 & 0.7545 \\
3 & $T_{1}-S_{2}-D_{1}-I_{1}-T_{1}$ & 4 & 0.3165 & 0.7287 \\
4 & $T_{1}-S_{1}-D_{1}-I_{1}-T_{1}$ & 0.3188 & 0.7270 \\
5 & $T_{1}-S_{2}-D_{2}-I_{1}-T_{1}$ & 4 & 0.3550 & 0.7011 \\
6 & $T_{1}-S_{2}-D_{1}-I_{2}-T_{1}$ & 4 & 0.3669 & 0.6929 \\
7 & $T_{1}-S_{2}-D_{2}-I_{2}-T_{1}$ & 0.3669 & 0.6929 \\
8 & $T_{1}-S_{2}-D_{1}-D_{2}-I_{1}-T_{1}$ & 4 & 0.3679 & 0.6922 \\
9 & $T_{1}-S_{1}-D_{1}-I_{2}-T_{1}$ & 0.3696 & 0.6910 \\
10 & $T_{1}-S_{2}-D_{1}-D_{2}-I_{2}-T_{1}$ & 0.3777 & 0.6854 \\
11 & $T_{1}-S_{2}-D_{2}-D_{1}-I_{1}-T_{1}$ & 5 & 0.3824 & 0.6822 \\
12 & $T_{1}-S_{2}-D_{2}-D_{1}-D_{2}-I_{1}-T_{1}$ & 4 & 0.6575 \\
13 & $T_{1}-S_{2}-D_{2}-D_{1}-D_{2}-I_{2}-T_{1}$ & 5 & 0.4191 & 0.6515 \\
14 & $T_{1}-S_{2}-D_{2}-D_{1}-I_{2}-T_{1}$ & 6 & 0.4284 & 0.6503 \\
15 & $T_{1}-S_{1}-D_{2}-D_{1}-D_{2}-I_{1}-T_{1}$ & 6 & 0.4304 & 0.6401 \\
16 & $T_{1}-S_{1}-D_{2}-D_{1}-D_{2}-I_{2}-T_{1}$ & 5 & 0.4461 & 0.6338 \\
\hline
\end{tabular}

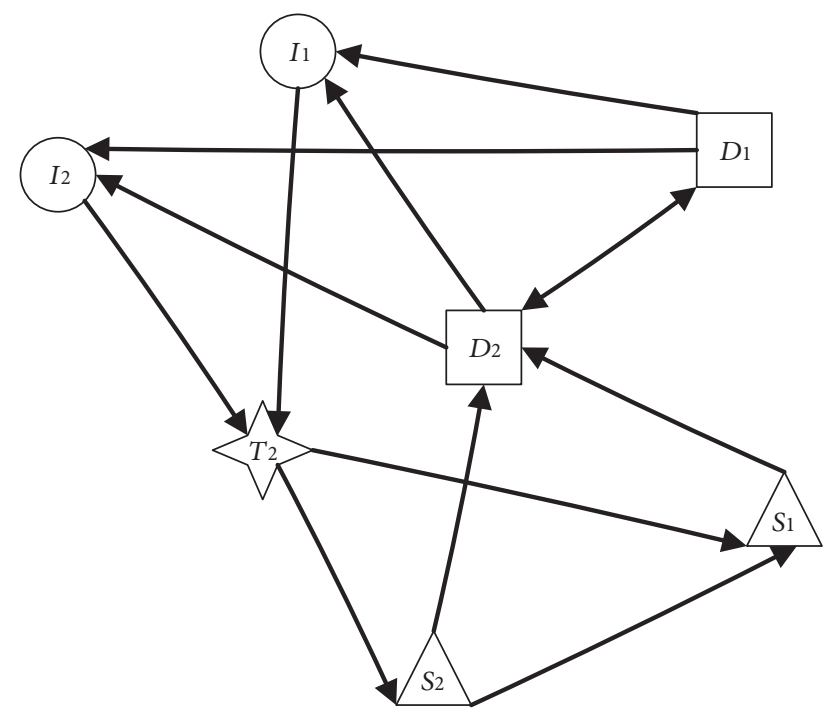

Figure 5: MAV/UAV collaborative combat network aimed at target $T_{2}$.

combat aircraft $I_{1}$ is highly similar to that of the unmanned combat aircraft. Therefore, considering such factors as the manned aircraft cost and pilot safety, an unmanned combat aircraft should be selected as much as possible to execute missions.

(2) Influence of the operation loop length on the combat capability: the observations listed in Tables 6 and 7 indicate that the length of the operation loop has a negative relation to its combat capability, which means that a longer operation loop has a lower combat capability. Each equipment in the operation loop brings a time delay, which becomes longer with more equipment, thereby influencing the combat capability of the overall operation loop.

(3) Influence of collaborative combat on combat capability: the length of operation loops 1 and 2 is greater than that of 3 and 4 . Thus, according to the previous conclusion, the combat capability of operation loops 1 and 2 should be inferior to that of 3 and 4; however, the opposite is true. The reason is that during the detection of target $T_{1}$ with 1 and 2, unmanned reconnaissance aircraft $S_{2}$ transmits its information to manned aircraft $S_{1} . S_{1}$ conducts reconnaissance of an enemy target and integrates information from both sides, which significantly reduces the information uncertainty of target $T_{1}$. Thus, the combat capability of 1 and 2 does not decrease due to the greater operation loop length but instead increases, which reflects the emergent properties and synergy of the combat systems.

\section{Conclusion}

This work models an MAV/UAV collaborative combat system network with a formal representation of the nodes and edges in the system. A capability assessment method and process for collaborative combat is introduced based on information entropy. The combat network modeling and corresponding capability assessment methods are verified by examples. The information entropy and operational capability of the collaborative combat network are calculated, the final combat capability evaluation index is obtained, and the operation loop capability is analyzed. The suggested method allows for a fast and reliable evaluation of the combat capabilities of different MAV/UAV systems as well as for the rapid 
TABLE 7: Energy and length of operation loops aimed at target $T_{2}$.

\begin{tabular}{lcccc}
\hline Number & Operation loop & Number of nodes & Length of operation loop & Capability of operation loop \\
\hline 1 & $T_{2}-S_{2}-S_{1}-D_{2}-I_{1}-T_{2}$ & 5 & 0.1693 & 0.8442 \\
2 & $T_{2}-S_{2}-S_{1}-D_{2}-I_{2}-T_{2}$ & 5 & 0.2091 & 0.8113 \\
3 & $T_{2}-S_{2}-D_{2}-I_{2}-T_{2}$ & 4 & 0.2275 & 0.7966 \\
4 & $T_{2}-S_{1}-D_{2}-D_{1}-I_{1}-T_{2}$ & 5 & 0.2375 & 0.7886 \\
5 & $T_{2}-S_{1}-D_{2}-I_{2}-T_{2}$ & 0.2548 & 0.7751 \\
6 & $T_{2}-S_{2}-D_{2}-D_{1}-D_{2}-I_{1}-T_{2}$ & 4 & 0.2613 & 0.7701 \\
7 & $T_{2}-S_{2}-S_{1}-D_{2}-D_{1}-D_{2}-I_{1}-T_{2}$ & 6 & 0.2308 & 0.757 \\
8 & $T_{2}-S_{1}-D_{2}-D_{1}-D_{2}-I_{1}-T_{2}$ & 7 & 0.2818 & 0.7544 \\
9 & $T_{2}-S_{2}-D_{2}-D_{1}-D_{2}-I_{2}-T_{2}$ & 6 & 0.3115 & 0.7324 \\
10 & $T_{2}-S_{1}-D_{2}-D_{1}-I_{2}-T_{2}$ & 6 & 0.3215 & 0.7251 \\
11 & $T_{2}-S_{2}-S_{1}-D_{2}-D_{1}-D_{2}-I_{2}-T_{2}$ & 5 & 0.2751 & 0.7235 \\
12 & $T_{2}-S_{1}-D_{2}-D_{1}-D_{2}-I_{2}-T_{2}$ & 7 & 0.3359 & 0.7147 \\
\hline
\end{tabular}

identification of the key assets and issues that can influence the combat capabilities of such systems. The results of this research are expected to be supplementary to previous research. The new method is useful to the development of collaborative combat system.

\section{Data Availability}

The data used to support the findings of this study are available from the corresponding author upon request.

\section{Conflicts of Interest}

The authors declare that they have no conflicts of interest.

\section{References}

[1] X. P. Zhu and Z. Zhou, "Development and outlook of the tactical unmanned air vehicle," Flight Dynamics, vol. 23, no. 2, pp. 1-4, 2005.

[2] W. Li and J. Chen, "Review and prospect of cooperative combat of manned/unmanned aerial vehicle hybrid formation," Aerospace Control, vol. 35, no. 3, pp. 90-96, 2017.

[3] H. Liu, X. Wei, Z. Fu, and Z. Zhou, "Cooperative task assignment of manned/unmanned aerial vehicle formation in air combat," Electronics Optics \& Control, vol. 20, no. 6, pp. 1619, 2013.

[4] X. L. Ma, Y. Y. Lei, Y. Q. Sun, and D. X. Liu, "Key technologies for cooperation of MAV/UAVs in air to ground attaching," Electronics Optics \& Control, vol. 18, no. 3, pp. 56-60, 2011.

[5] Department of Defense Architecture Framework Group, Department of Defense Architecture Framework Version 2.0, DoDAF, Washington D. C, 2009.

[6] T. R. Ender, A Top-Down Hierarchical System-of-Systems Approach to the Design of an Air Defense Weapon, Georgia Institute of Technology, Atlanta, 2006.

[7] A. Aaron and B. Bazin, "Boyd's OODA loop and the infantry company commander," Infantry, vol. 18, no. 1, pp. 17-19, 2005.
[8] J. R. Cares and Alidade Incorporated, "An information age combat model," in 9th International Command and Control Research and Technology Symposium, pp. 1-27, Copenhagen, Denmark, 2004.

[9] J. R. Cares, Distributed Networked Operations: the Foundations of Network Centric Warfare, IUniverse, 2006.

[10] A. H. Dekker, "Network topology and military performance," in MODSIM 2005 International Congress on Modelling and Simulation, Modelling and Simulation Society of Australia and New Zealand, A. Zerger and R. M. Argent, Eds., pp. 2174-2180, 2005.

[11] A. Tolk, S. Deller, M. I. Bell, S. R. Bowling, G. A. Rabadi, and R. Huber, "Applying the information age combat model: quantitative analysis of network centric operations," International C2 Journal, vol. 3, no. 1, pp. 1-25, 2009.

[12] W. X. Jin, Modeling and Simulation of System-of-Systems Combat Complex Network, Electronics Industry Press, Beijing, 2010.

[13] J. Q. Li, G. G. Liu, Q. Huang, Y. F. Cai, and X. Z. Zeng, “Analysis method and model of combat command architecture based on theory of complex networks," Journal of System Simulation, vol. 20, no. 17, pp. 4712-4715, 2008.

[14] B. X. Jiang and H. F. Hao, "Combat capability of the army based on information entropy theory," Journal of Ordnance Equipment Engineering, vol. 31, no. 6, pp. 113-114, 2010.

[15] J. Xu and S. Zhang, "Research on integrative operation effectiveness evaluation of anti-missile systems based on information entropy," Command Control \& Simulation, vol. 32, no. 5, pp. 63-66, 2010.

[16] W. Perry, R. W. Button, J. Bracken, T. Sullivan, and J. Mitchell, Measurements of Effectiveness for the Information-Age Navy: The Effects of Network-Centric Operations on Combat Outcomes, Rand, 2002.

[17] H. W. Liu, A Study on Feature Selection Algorithms Using Information Entropy, Jilin University, 2010. 


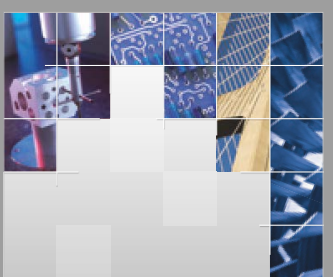

\section{Enfincering}
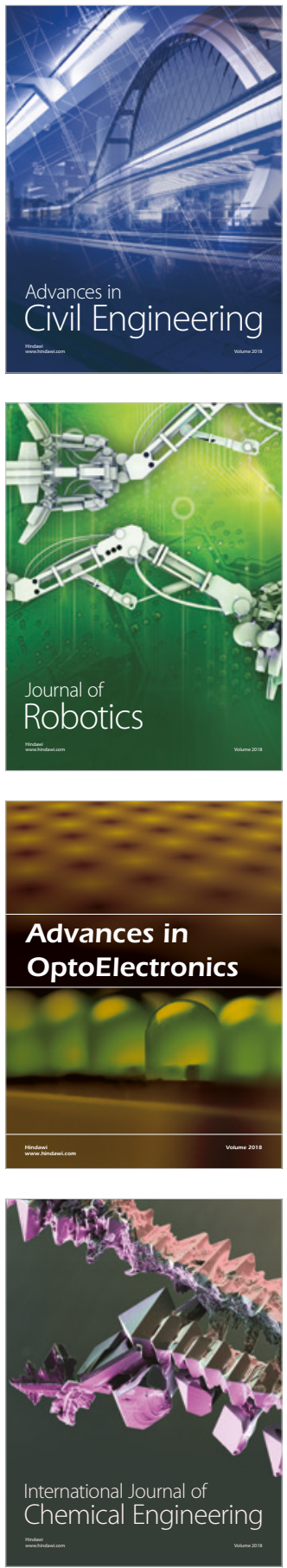

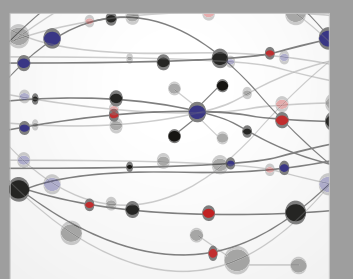

\section{Rotating \\ Machinery}

The Scientific World Journal

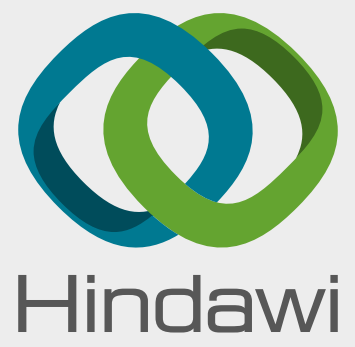

Submit your manuscripts at

www.hindawi.com
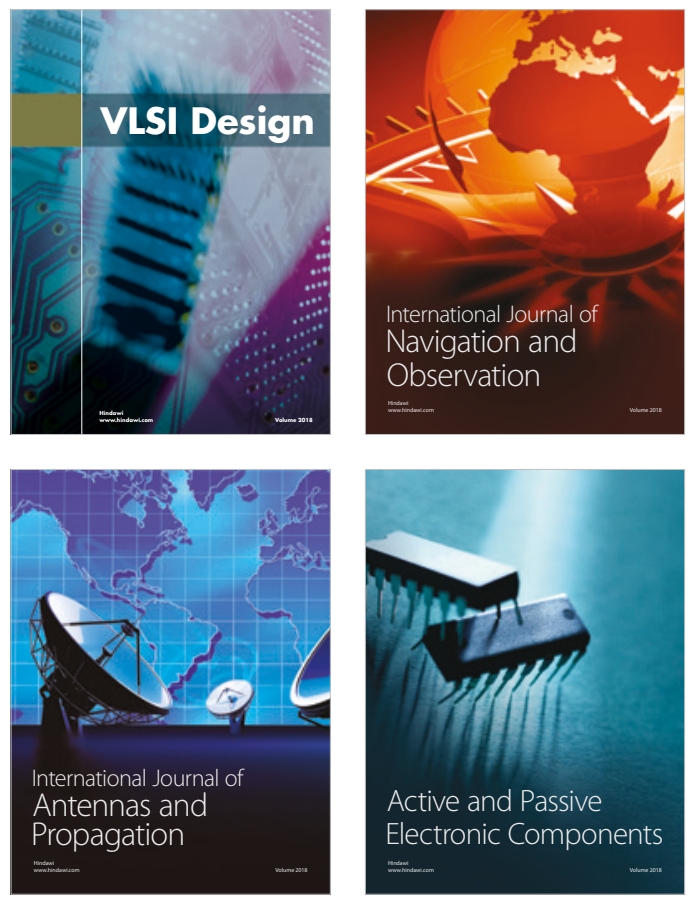
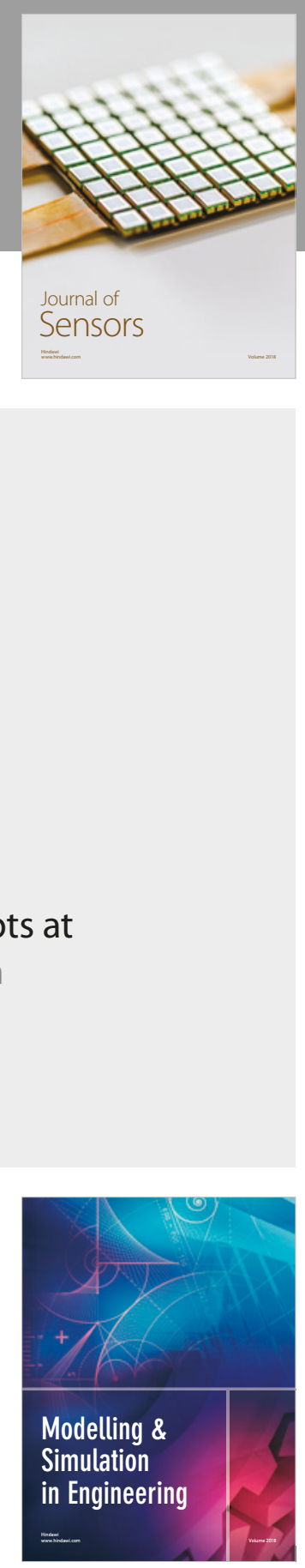

\section{Advances \\ Multimedia}
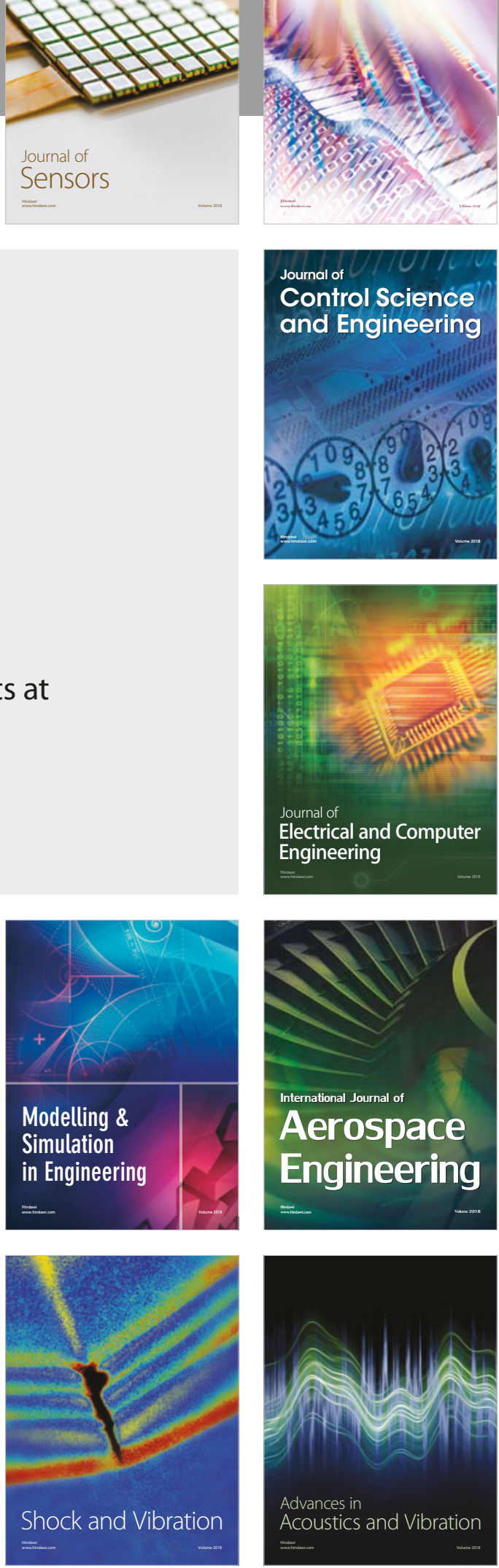